\title{
RELAÇÕES DE APORTE DE SEDIMENTO E IMPLICAÇÕES DE SUA UTILIZAÇÃO NO PAGAMENTO POR SERVIÇO AMBIENTAL EM BACIAS HIDROGRÁFICAS ${ }^{(1)}$
}

\author{
Henrique Marinho Leite Chaves ${ }^{(2)}$
}

\begin{abstract}
RESUMO
Em razão de sua simplicidade, a relação de aporte de sedimento (RAS), dada pela razão entre a produção de sedimento no exutório e a erosão total na bacia, é comumente usada no estudo e na determinação da produção de sedimentos em bacias hidrográficas. Como há uma série de complexidades ligando a produção de sedimentos (Y) e a erosão a montante (E) na bacia, foram desenvolvidas distintas equações de RAS. Programas de pagamento por serviço ambiental relativos ao abatimento de sedimentação, como o Produtor de Água, frequentemente requerem a estimativa da redução da produção de sedimentos nas bacias hidrográficas, em função de implantação do controle de erosão a montante. Os objetivos do presente trabalho foram calcular a RAS da bacia do ribeirão Pipiripau (DF), usando oito equações existentes na literatura, e estimar a provável redução em Y, em função de uma redução de 50 \% da erosão na bacia, bem como avaliar as eventuais dificuldades em sua aplicação. Os valores de RAS obtidos variaram entre 0,12 e 0,52, com média de 0,24 e desvio-padrão de 0,14. Duas das equações apresentaram alta sensibilidade às variáveis, indicando que haveria significativo potencial de propagação de erro se elas fossem usadas. Partindo-se de uma redução de erosão de $50 \%$ na bacia (117.500 t ano-1), a redução no aporte de sedimento usando as RAS

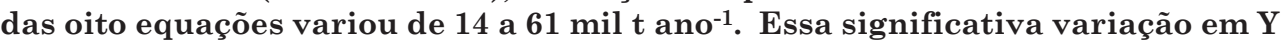
indica considerável incerteza na estimativa de serviços ambientais relativos à redução de aporte de sedimento, em programas de controle de erosão.
\end{abstract}

Termos de indexação: potencial de erosão, gestão pequenas bacias, RAS, serviço ambiental.

\footnotetext{
(1) Recebido para publicação em setembro de 2008 e aprovado em maio de 2010.

(2) Professor de Manejo de Bacias Hidrográficas. Departamento de Engenharia Florestal, Faculdade de Tecnologia - UnB. Caixa Postal 04357, CEP 70.910-900 Brasília (DF). E-mail: hchaves@unb.br
} 


\title{
SUMMARY: RELATIONS OF SEDIMENT DELIVERY UNDERLYING PAYMENTS FOR ENVIRONMENTAL SERVICES IN CATCHMENT AREAS
}

\begin{abstract}
Because of its simplicity, the sediment delivery ratio (SDR), based on the ratio of the sediment yield $(Y)$ and the gross erosion $(E)$ in the basin, is widely used to estimate sediment production in watersheds. Since Y and $E$ are complexly linked, several SDR equations were developed. Financial compensation programs resulting from sedimentation abatement, such as the Water Provider Program, often require the estimation of reduction of the downstream sediment yield as a function of erosion reduction upstream. This study was conducted at the Forestry Department of the Universidade de Brasilia-UnB, and in the Pipiripau river basin, in November 2007. The SDR of the Pipiripau river basin (DF) was calculated based on eight equations used in the literature, to estimate the reduction in $Y$ due to a reduction of $50 \%$ of $E$ in the basin, as well as to evaluate eventual limitations of their application. The SDR values varied from 0.12 to 0.52 , with a mean of 0.24 and a standard deviation of 0.14 . Two of the eight equations were highly sensitive to the variables, indicating a high potential for error propagation. With a $50 \%$ reduction in the gross upstream erosion (117.500 tyr $\left.{ }^{-1}\right)$, the corresponding reduction in sediment yield using the SDR of the eight equations varied from 14,000 to 61,000 $t \mathrm{yr}^{-1}$. This significant variation in sediment yield results in uncertainties in the estimation of the environmental services for sedimentation abatement, in erosion control programs.
\end{abstract}

Index terms: erosion potential, watershed management, SDR, environmental services.

\section{INTRODUÇÃO}

O pagamento por serviços ambientais (PSA) vem se tornando uma ferramenta interessante em iniciativas de controle da erosão e da sedimentação em pequenas bacias hidrográficas rurais (Young, 2005). Dentre elas, destaca-se o Programa do Produtor de Água (Chaves et al., 2004a,b), que já vem sendo implementado em algumas bacias piloto no Brasil (PCJ, 2009). Esse programa utiliza, para avaliação do serviço ambiental relativo ao abatimento da erosão, uma forma simplificada da USLE (Wischmeier \& Smith, 1978), a fim de facilitar sua aplicação por produtores e agentes de extensão rural.

Nesse programa, os fatores C e P da USLE, estimados para as condições de uso e manejo e práticas conservacionistas, antes e depois da implantação do projeto, são usados no cálculo do abatimento relativo da erosão na gleba, supondo-se que os outros fatores permaneçam constantes. Assim, o percentual de abatimento de erosão após a implantação do projeto é calculado por meio da seguinte equação (Chaves et al., 2004a):

$$
A_{e}=100\left(1-\frac{Z_{1}}{Z_{2}}\right)
$$

em que $A_{e}(\%)=$ abatimento relativo da erosão na gleba $; \mathrm{Z}_{0}=\mathrm{C}_{0} \mathrm{P}_{0} ; \mathrm{Z}_{1}=\mathrm{C}_{1} \mathrm{P}_{1}$, sendo $\mathrm{C}_{1}$ (adim.) $=$ fator de uso e manejo do solo depois da implantação do projeto; $\mathrm{P}_{1}$ (adim.) = fator de práticas conservacionistas depois da implantação do projeto; $\mathrm{C}_{0}=$ fator de uso e manejo do solo antes da implantação do projeto; e $\mathrm{P}_{0}$ (adim.) $=$ fator de práticas conservacionistas antes da implantação do projeto.

A filosofia do programa é que a redução da erosão implicará redução proporcional na produção de sedimentos no exutório da bacia, beneficiando diferentes usuários de água e o meio ambiente (Chaves et al., 2004b).

A redução do aporte de sedimento, por sua vez, pode ser estimada pela relação de aporte de sedimento (RAS), largamente empregada em estudos sedimentológicos. Essa relação é dada pela seguinte expressão (Renfro, 1975; Walling, 1983; Lu et al., 2006):

$$
R A S=\frac{Y}{E}
$$

em que RAS $(0-1)=$ relação de aporte de sedimento; $Y$ $(\mathrm{t})=$ produção de sedimento no exutório da bacia; e E (t) erosão total na bacia. Y e E podem ser calculados anualmente $\left(\mathrm{t} \mathrm{ano}^{-1}\right)$ ou por unidade de área $\left(\mathrm{t} \mathrm{km}^{-2}\right)$ (Lu et al., 2006).

Considerando que a RAS é constante para uma dada bacia em um determinado tempo (Walling, 1983), conhecendo-se a erosão total (E) e sua redução com a implantação de programas de controle de erosão $(\Delta \mathrm{E})$, bem como a RAS da bacia, podem-se estimar os valores de produção de sedimento no exutório $(\mathrm{Y})$ e sua redução $(\Delta \mathrm{Y})$. A redução $\Delta \mathrm{Y}$, por sua vez, pode ser usada como indicador de serviço ambiental gerado, para posterior compensação financeira aos produtores participantes (Chaves \& Piau, 2008). 
Entretanto, apesar da simplicidade da equação (2), há uma série de complexidades ligando o aporte de sedimento a jusante com a perda de solo a montante (Walling, 1988). De acordo com esse autor, o valor da RAS é influenciado por uma variedade de fatores geomorfológicos e ambientais, incluindo a natureza, a extensão e a localização das fontes de sedimento, as características do relevo, o padrão de drenagem da bacia e as condições dos canais, bem como a condição de cobertura vegetal, uso da terra e textura do solo.

Apesar dessa complexidade, a RAS é frequentemente estimada por equações empíricas, em que a área da bacia é uma das variáveis independentes mais usadas (Renfro, 1975; Walling, 1988). Expressões típicas relacionando o RAS e a área da bacia são geralmente da seguinte forma (Lu et al., 2006):

$$
R A S=a \mathrm{~A}^{-\mathrm{b}}
$$

em que $\mathrm{A}\left(\mathrm{km}^{2}\right)=$ área da bacia; $a$ e $b=$ parâmetros empíricos de ajuste, dependentes das características físicas e hidrológicas da bacia. Lu et al. (2006) encontraram valores de $b$ entre 0,01 e 0,25 . O valor negativo de $b$ na equação (3) indica que, com o aumento da área, há redução no RAS. Isso é explicado pelo fato de que, com o aumento da área da bacia, há redução da declividade das vertentes e do gradiente do canal principal, aumentando as chances de deposição no interior da bacia (Walling, 1988).

Renfro (1975), estudando o comportamento sedimentológico de 14 pequenas bacias do Texas (EUA), com áreas variando entre 1,0 e $262 \mathrm{~km}^{2}$, concluiu que a RAS variou de 0,67 a 0,26 , respectivamente.

Além da área da bacia, outras variáveis independentes são usadas para estimativa de RAS. Estas incluem o gradiente do canal principal (Williams $\&$ Berndt, 1972), o relevo $(\Delta \mathrm{H})$ da bacia (Maner, 1958; Roehl, 1962), a relação de bifurcação da rede de drenagem (Roehl, 1962), o coeficiente de escoamento (Williams, 1977) e os tempos de concentração e de duração da chuva em excesso (Lu et al., 2006).

Entretanto, a falta de uma equação de RAS universalmente aplicável deve-se em parte à complexidade do processo sedimentológico e sua interação com as características da bacia, além da falta de levantamentos exatos da produção de sedimento e da erosão total (Walling, 1988). Por outro lado, é fundamental que a RAS seja obtida da forma mais acurada possível, de modo que a produção de sedimento no exutório da bacia, raramente disponível em pequenas bacias, possa ser calculada com precisão (Ouyang \& Bartholic, 1997).

No Brasil, há muitos estudos de perda de solo em vertentes, mas poucas informações a respeito da produção de sedimento em bacias. Entretanto, uma vez obtidos valores confiáveis de RAS para essas bacias, podem-se obter resultados mais precisos do aporte de sedimento, e de sua redução, por meio da implantação de programas conservacionistas.
Souza et al. (2006) usaram as equações de Vanoni (1975) e de Williams \& Berndt (1972) para estimar o aporte de sedimento em uma pequena bacia do Rio Grande do Sul. Os valores de RAS obtidos foram de 0,21 e 0,063 , respectivamente. Como consequência, o aporte de sedimento calculado pela primeira foi três vezes maior que o da segunda.

Silva (2001), usando um expoente de 0,2 para a equação (3) para obter o aporte de sedimento na bacia do rio Paracatu, a partir da estimativa da erosão total na bacia com a USLE, concluiu que Y calculado pela equação (2) superestimou o aporte médio anual de sedimentos em suspensão observado em $276 \%$.

Considerando o exposto, o objetivo do presente trabalho foi estimar a relação de aporte de sedimento (RAS) para uma pequena bacia hidrográfica rural do Distrito Federal, candidata à implantação de um programa de controle da erosão e sedimentação, usando diferentes equações de RAS existentes na literatura, bem como analisar as implicações relativas ao uso dessas equações na estimativa do aporte de sedimento resultante.

\section{MATERIAL E MÉTODOS}

\section{Equações de aporte de sedimento analisadas}

Oito equações de cálculo da relação de aporte de sedimento (RAS) existentes na literatura foram usadas neste estudo (Quadro 1).

A maioria das equações do quadro 1 foi desenvolvida em pequenas bacias hidrográficas dos EUA, porém validadas em diferentes regiões do mundo. Oito diferentes variáveis fisiográficas e hidrológicas são usadas para a estimativa da RAS (Quadro 1), o que permitiu um exame bastante amplo de sua variação.

\section{Características físicas e hidrológicas da bacia estudada}

A bacia estudada foi a do ribeirão Pipiripau, localizada na região nordeste do Distrito Federal. A bacia, de uso dominantemente rural, tem uma área de $235 \mathrm{~km}^{2}$ e apresenta relevo suave ondulado. Os solos dominantes são Latossolos argilosos, com presença de Cambissolos e Neossolos Quartzarênicos (CAESB, 2001).

De acordo com a classificação de Köppen, o clima da região é Aw, com precipitação pluvial média anual de $1.306 \mathrm{~mm}$. A vazão média de longo termo do ribeirão Pipiripau é de $2,89 \mathrm{~m}^{3} \mathrm{~s}^{-1}$ (Chaves \& Piau, 2008).

Nas figuras 1, 2 e 3 são apresentados os principais solos, os tipos de uso do solo e a declividade das vertentes da bacia do ribeirão Pipiripau, respectivamente. 
Quadro 1. Equações de relação de Aporte de Sedimentos (RAS) usadas no trabalho, com os respectivos autores e variáveis

\begin{tabular}{|c|c|c|}
\hline Autor (ano) & Equação & Descrição das variáveis \\
\hline Maner (1958) & $\begin{array}{l}\log \text { RAS }=2,962+0,869 \log R \\
-0,854 \log L\end{array}$ & $\begin{array}{l}\mathrm{R}=\text { diferença de altura entre ponto mais alto e exutório }(\mathrm{m}) \\
\mathrm{L}=\text { comprimento da bacia }(\mathrm{m})\end{array}$ \\
\hline Roehl (1962) & $\begin{array}{l}\log \operatorname{RAS}=4,5-0,23 \log \mathrm{A} \\
-0,51 \operatorname{colog}(\mathrm{R} / \mathrm{L})-2,79 \log \mathrm{B}_{\mathrm{r}}\end{array}$ & $\begin{array}{l}\mathrm{A}=\text { área da bacia }\left(\mathrm{km}^{2}\right) \\
\mathrm{R}=\text { diferença de altura entre ponto mais alto e exutório }(\mathrm{m}) \\
\mathrm{L}=\text { comprimento da bacia }(\mathrm{m}) \\
\mathrm{R}_{\mathrm{b}}=\text { relação de bifurcação de Horton }\end{array}$ \\
\hline Williams \& Berndt (1972) & $\operatorname{RAS}=0,627 \mathrm{D}^{0,403}$ & $\mathrm{D}=$ gradiente do canal principal $(\%)$ \\
\hline Renfro (1975) & $\log \operatorname{RAS}=1,793-0,142 \log \mathrm{A}$ & $\mathrm{A}=$ área da bacia $\left(\mathrm{km}^{2}\right)$ \\
\hline Vanoni (1975) & $\mathrm{RAS}=0,42 \mathrm{~A}^{-0,125}$ & $\mathrm{~A}=$ área da bacia $\left(\mathrm{mi}^{2}\right)^{(1)}$ \\
\hline Williams (1977) & $\begin{array}{l}\mathrm{RAS}=1,37 \times 10^{-11} \mathrm{~A}^{-0,00998} \\
(\mathrm{R} / \mathrm{L})^{0,363} \mathrm{CN}^{5,44}\end{array}$ & $\begin{array}{l}\mathrm{A}=\text { área da bacia }\left(\mathrm{km}^{2}\right) \\
\mathrm{R}=\text { diferença de altura entre ponto mais alto e exutório }(\mathrm{m}) \\
\mathrm{L}=\text { comprimento da bacia }(\mathrm{km}) \\
\mathrm{CN}=\text { número }- \text { curva do NRCS }\end{array}$ \\
\hline NRCS (1979) & $\operatorname{RAS}=0,51 \mathrm{~A}^{-0,11}$ & $\mathrm{~A}=$ área da bacia $\left(\mathrm{mi}^{2}\right)^{(1)}$ \\
\hline Lu et al. (2006) & $\begin{array}{l}\operatorname{RAS}=2\left(t_{r} / t_{c}\right)\left\{1-\left(t_{r} / t_{c}\right)+\left(t_{r} / t_{c}\right)\right. \\
\left.\exp \left[\left(t_{c} / t_{r}\right)\right]\right\}\end{array}$ & $\begin{array}{l}\mathrm{t}_{\mathrm{r}}=\text { duração do excesso de precipitação }(\mathrm{h}) \\
\mathrm{t}_{\mathrm{c}}=\text { tempo de concentração da bacia }(\mathrm{h})\end{array}$ \\
\hline
\end{tabular}

(1) mi: milhas terrestres.

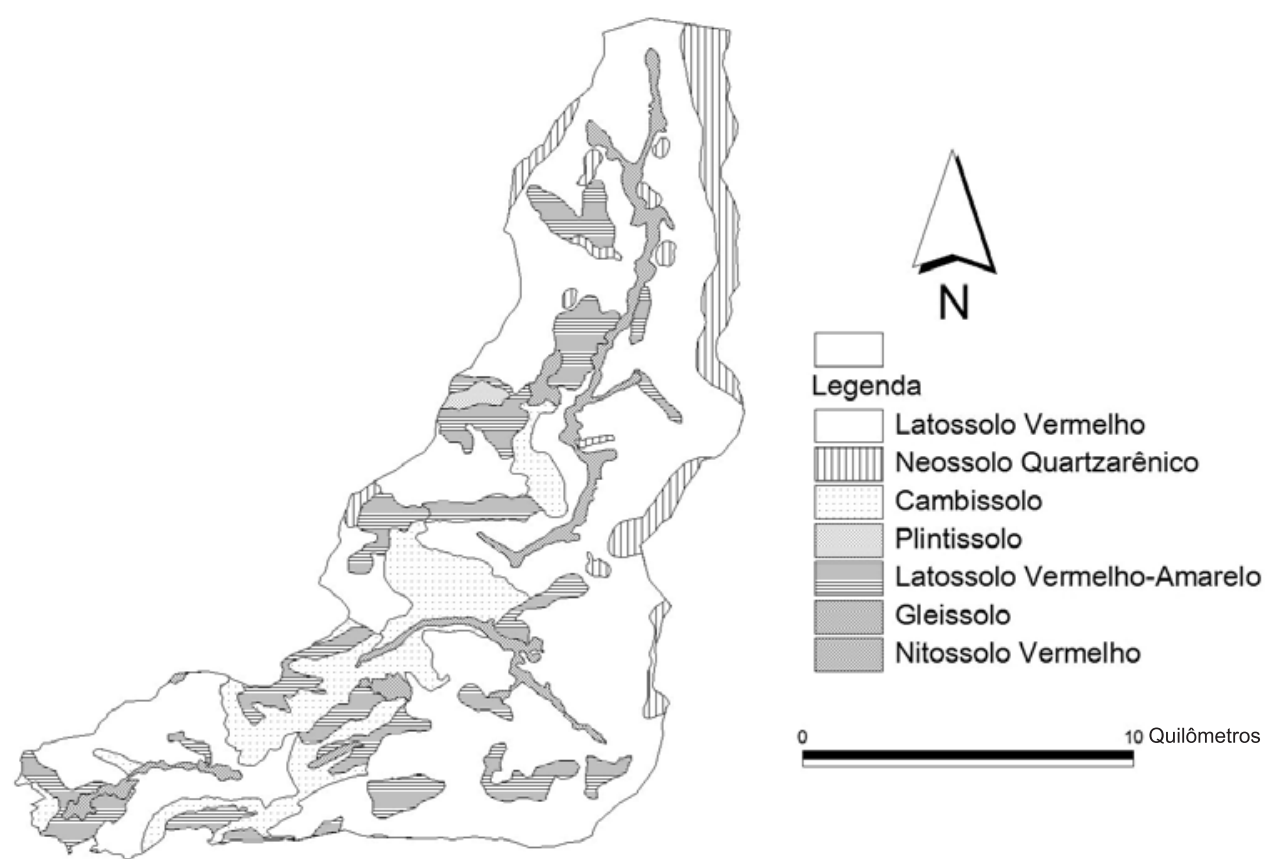

Figura 1. Principais classes de solos da bacia do ribeirão Pipiripau. Fonte: CAESB (2001).

Os Latossolos cobrem a maior parte $(74 \%)$ da bacia (Figura 1). A agricultura, pastagem e outros usos antrópicos constituem a maioria dos usos do solo da bacia (68 \% da área total, sendo $39 \%$ de agricultura), com $32 \%$ cobertos por vegetação nativa (Cerrado) (Figura 2).

A bacia apresenta relevo dominante plano a suave ondulado $\left(<5^{\circ}\right)$, cobrindo $58 \%$ da sua área total (Figura 3). Apesar de haver duas pequenas barragens de captação no canal principal do curso d'água, o fluxo do ribeirão Pipiripau pode ser considerado como a fio d’água.

A despeito de não haver dados sedimentológicos obtidos de maneira sistemática na bacia, um estudo prospectivo de produção de sedimento, realizado por meio de modelagem matemática, concluiu que o aporte de sedimento médio anual em seu exutório é de 25 mil toneladas (Chaves \& Piau, 2008). Esse valor representa uma produção específica de sedimento de $106 \mathrm{t} \mathrm{km}^{-2}$ ano $^{-1}$, considerada como moderada (Lima et al., 2001). 


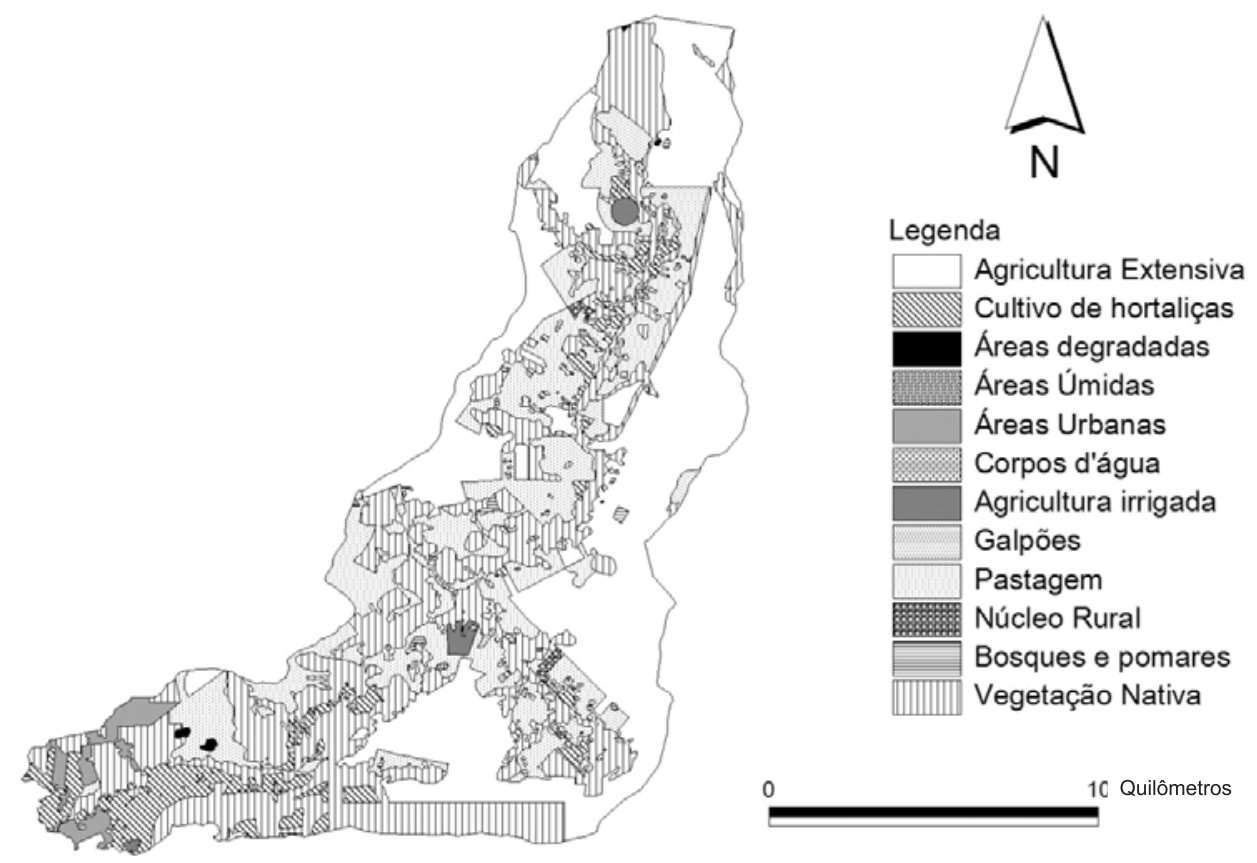

Figura 2. Tipos de uso do solo da bacia do ribeirão Pipiripau. Fonte: CAESB (2001).
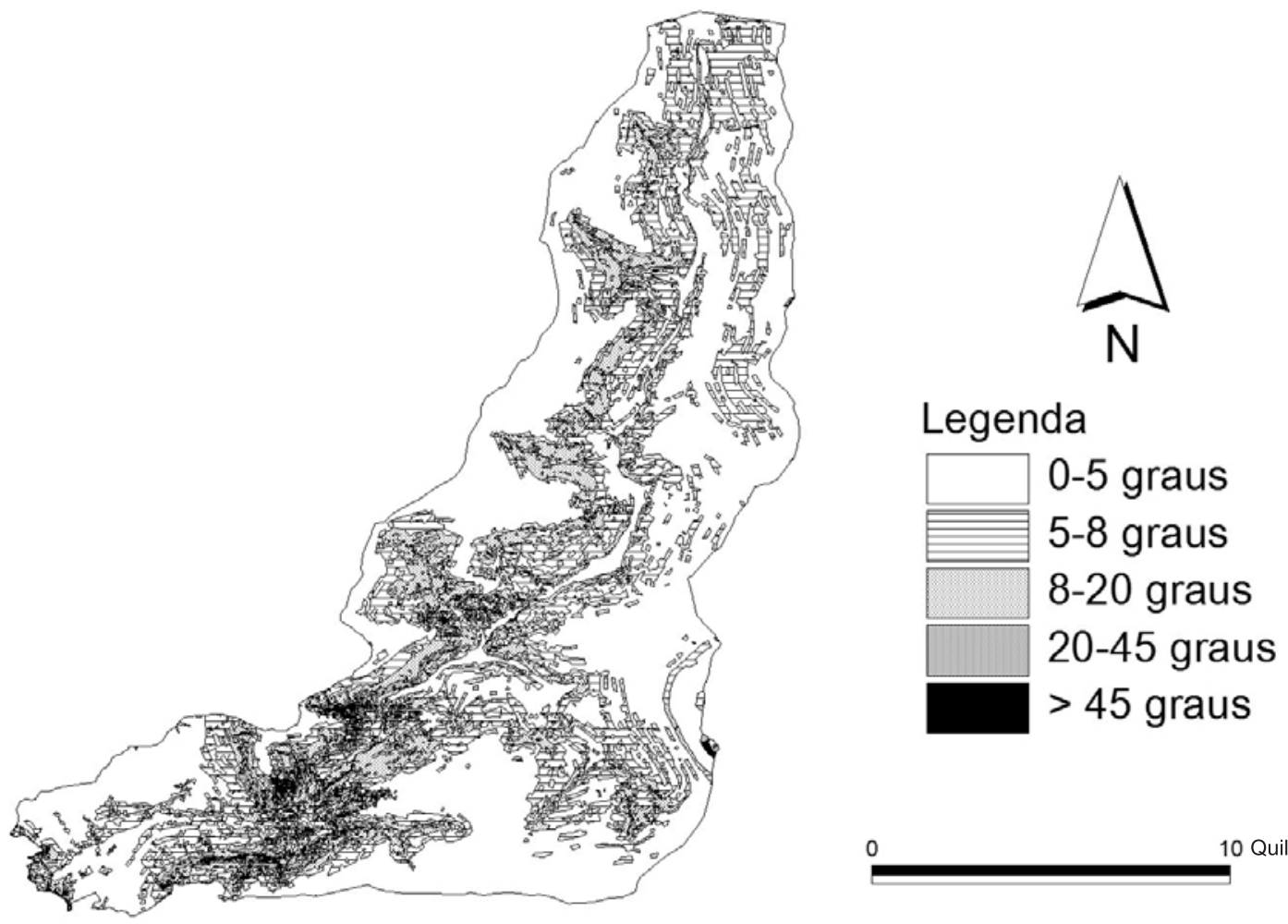

Figura 3. Declividade das vertentes da bacia do ribeirão Pipiripau. Fonte: CAESB (2001).

Chaves et al. (2004b), estudando o efeito da mudança do manejo do solo na bacia do ribeirão Pipiripau, da agricultura convencional para plantio direto, bem como a recuperação de pastagens degradadas, concluíram que elas permitiriam redução média de $73 \%$ na perda de solo na bacia. O quadro 2 apresenta os valores do fator $\mathrm{Z}$ da equação (1) antes e depois da implantação de um programa conservacionista em $100 \%$ das glebas com agricultura e pecuária na bacia. 
Quadro 2. Valores de $\mathrm{Z}$ para os diferentes tipos de uso da bacia do ribeirão Pipiripau e o do abatimento de erosão $\left(A_{e}\right)$ que seria obtido com a implantação de um programa de controle de erosão na bacia

\begin{tabular}{lrrrr}
\hline Tipo de uso & \% da bacia & $\mathbf{Z}_{\mathbf{0}}$ & $\mathbf{Z}_{\mathbf{1}}$ & $\mathbf{A}_{\mathbf{e}}$ \\
\hline Agricultura & 41,5 & 0,25 & 0,03 & $88 \%$ \\
Pastagem & 23,1 & 0,25 & 0,12 & $52 \%$ \\
Horticultura & 2,7 & 0,50 & 0,25 & $50 \%$ \\
Pomares & 0,7 & 0,37 & 0,11 & $70 \%$ \\
Total / Média & 68,0 & & & $73 \%$ \\
$\begin{array}{l}\text { Média considerando } \\
\text { todos os usos }\end{array}$ & & & & $50 \%$ \\
\hline
\end{tabular}

Fonte: Chaves et al. (2004b).

De acordo com o quadro 2, se um programa de controle de erosão fosse implantado na bacia do ribeirão Pipiripau, haveria redução média ponderada de $73 \%$ na erosão nas áreas de uso antrópico da bacia. Considerando a área total da bacia (em que 32 \% são cobertos por vegetação nativa ou áreas não erodíveis), a redução média ponderada da erosão seria de $50 \%$.

De acordo com a filosofia do Programa do Produtor de Água (Chaves et al., 2004a), o serviço ambiental desse tipo de redução seria sentido na redução do aporte de sedimento a jusante e na melhoria da qualidade da água da bacia. Para que esse benefício ambiental seja estimado, pode-se utilizar a RAS da bacia. Esse assunto é apresentado no item a seguir.

\section{Estimativa da RAS para a bacia do ribeirão Pipiripau}

As equações de RAS (Quadro 1) foram aplicadas na bacia do ribeirão Pipiripau. Para isso, os dados relativos às variáveis desse quadro foram levantados na bacia, por meio de análises espaciais no SIG (ESRIArcView $3.3 \AA$ ).

No caso do número-curva $(\mathrm{CN})$, o valor usado foi aquele resultante da calibração pela equação do volume de escoamento direto do NRCS (1972), em eventos individuais da bacia, conforme descrito por Chaves \& Piau (2008).
Já no caso do tempo de concentração da bacia $\left(t_{c}\right)$, este foi obtido pela equação de Kirpich (1940):

$$
\mathrm{t}_{\mathrm{c}}=0,0195 \mathrm{~L}^{0,77} \mathrm{D}^{-0,385}
$$

em que $t_{c}=$ tempo de concentração da bacia, definido como o tempo necessário durante uma chuva para que todos os pontos da bacia contribuam para sua vazão (min); L = comprimento máximo do escoamento (m); $\mathrm{e} \mathrm{D}=$ gradiente da bacia $\left(\mathrm{m} \mathrm{m}^{-1}\right)$. Apesar de a equação de Kirpich (1940) apresentar limitações relativas à não incorporação do tipo de uso do solo, ela possui poucos parâmetros e apresenta boa correlação com dados medidos (Magalhães, 1989).

A duração da chuva em excesso ( $t_{\mathrm{r}}$ ) foi tomada como sendo $t_{r}=0,5$, conforme sugerido por Lu et al. (2006), obtido a partir de médias de séries de dados hidrológicos.

A relação de bifurcação de redes de drenagem $\left(R_{b}\right)$, por sua vez, foi estimada pela seguinte equação (Chow et al., 1988):

$$
R_{b}=\frac{N_{i}}{N_{i+1}}
$$

em que $\mathrm{N}_{\mathrm{i}}=$ número de canais de ordem i; e $\mathrm{N}_{\mathrm{i}+1}=$ número de canais de ordem $\mathrm{i}+1$.

De posse dos valores das variáveis de RAS para a bacia (Quadro 3), as equações do quadro 1 foram usadas para calcular oito valores de RAS. Desses valores, foram computados a média, o desvio-padrão e o coeficiente de variação.

Em seguida, foi feita uma análise de sensibilidade da RAS a cada uma das variáveis do quadro 3. Assim, a sensibilidade relativa $\left(S_{r}\right)$ da RAS a uma pequena variação em uma certa variável $x$ foi calculada pela seguinte equação (McCuen \& Snyder, 1986):

$$
S_{r}=\frac{\left(S D R_{j}-S D R_{i}\right) / S D R_{i}}{\left(x_{j}-x_{i}\right) / x_{i}}
$$

em que $\mathrm{S}_{\mathrm{r}}=$ sensibilidade relativa da RAS; $\mathrm{RAS}_{\mathrm{i}}=$ valor de RAS calculado de acordo com os valores originais das variáveis (Quadro 3); $\mathrm{x}_{\mathrm{i}}=$ nível original da variável i; $x_{j}=\left(x_{i}+\Delta x_{i}\right)$; e RAS $\mathrm{R}_{\mathrm{j}}=$ valor de RAS usando o valor acrescido $x_{j}$. No presente estudo, o valor da perturbação $\Delta x_{i}$ foi de $10 \%$ do valor original de $x_{i}$ (Chaves, 1991).

Quadro 3. Valores das variáveis utilizadas no cálculo da RAS, usando as equações do quadro 1

\begin{tabular}{cccccccc}
\hline Área & Compr. do Canal (L) & Desnível (R) & Gradiente (D) & $\mathbf{C N}^{(1)}$ & $\mathbf{R}_{\mathbf{b}}{ }^{(2)}$ & $\mathbf{t}_{\mathbf{c}}{ }^{(3)}$ & $\mathbf{t}_{\mathbf{r}}{ }^{(4)}$ \\
\hline $\mathrm{km}^{2}$ & $\mathrm{~km}$ & $\mathrm{~m}$ & $\%$ & & & - \\
235 & 34,7 & 215 & 0,62 & 68,2 & 4,3 & 7,2 & 0,5 \\
\hline
\end{tabular}

(1) Número-curva. ${ }^{(2)}$ Relação de Bifurcação da rede de drenagem. ${ }^{(3)}$ Tempo de concentração da bacia (h). ${ }^{(4)}$ Tempo de recessão da bacia (h). 


\section{Implicações do uso da RAS na estimativa do serviço ambiental relativo à sedimentação}

A partir da variabilidade observada nos valores de RAS das oito equações (Quadro 1) e da sensibilidade relativa de cada uma das equações às variáveis do quadro 3, foram feitas análises sobre a implicação de seu uso como estimador da produção de sedimentos no exutório da bacia do ribeirão Pipiripau.

Mais especificamente, foi avaliada a variação nos valores da produção de sedimentos $\mathrm{Y}$, em função de um abatimento de $50 \%$ da erosão na bacia $\left(\mathrm{A}_{\mathrm{e}}\right)$, conforme estimado por Chaves et al. (2004b) (Quadro 2).

Supondo que a erosão média nas glebas da bacia antes da implantação do controle da erosão seja de $10 \mathrm{t} \mathrm{ha}^{-1} \mathrm{ano}^{-1}$, um abatimento relativo médio ponderado de $\mathrm{A}_{\mathrm{e}}=50 \%$, conforme estimado por Chaves et al. (2004b) para a situação após a implantação do programa, resultaria em redução de perda de solo total na bacia de $\Delta \mathrm{E}=5,0 \mathrm{t} \mathrm{ha}^{-1} \mathrm{ano}^{-1} \times 23.500 \mathrm{ha}=$ $117.500 \mathrm{t} \mathrm{ano}^{-1}$.

Partindo da premissa de que a equação (2) é também válida para variações de $\mathrm{Y}$ e A e usando o valor de $\Delta \mathrm{E}$ indicado, foram obtidos valores de $\Delta \mathrm{Y}$ para cada uma das equações do quadro 1 , ou seja:

$$
\Delta Y=S D R . \Delta \mathrm{E}
$$

em que $\Delta Y=$ variação no aporte de sedimento da bacia $\left(\mathrm{t}\right.$ ano $\left.{ }^{-1}\right)$, resultante de uma variação na erosão total, $\Delta E\left(\mathrm{t} \mathrm{ano}^{-1}\right)$; e RAS, definida anteriormente.

\section{RESULTADOS E DISCUSSÃO}

\section{Relação de aporte de sedimentos}

Os valores de RAS (Figura 4) variaram entre 0,12 e 0,52, com média de 0,24 e desvio-padrão de 0,14, sendo este último bastante significativo (Figura 4). A equação que mais se aproximou do valor médio foi a de Vanoni (1975).

Renfro (1975) obteve experimentalmente um valor de RAS de 0,26 para uma bacia de $262 \mathrm{~km}^{2}$ no Texas (EUA) - muito próximo da média observada para a bacia do Pipiripau $\left(235 \mathrm{~km}^{2}\right)$ (Figura 1).

\section{Análise de sensibilidade das equações de RAS}

Na figura 5 é apresentada a sensibilidade média relativa das oito equações usadas no cálculo de RAS e suas variáveis, calculadas pela equação (6), e a figura 6 mostra a sensibilidade relativa máxima observada, ambas usando uma perturbação de $\Delta x_{i}=10 \% x_{i}$. Os valores das duas figuras correspondem aos módulos das sensibilidades relativas $\left(\left|\mathrm{S}_{\mathrm{r}}\right|\right)$. Na figura 6 , a variável que proporcionou a máxima sensibilidade relativa da RAS é identificada.

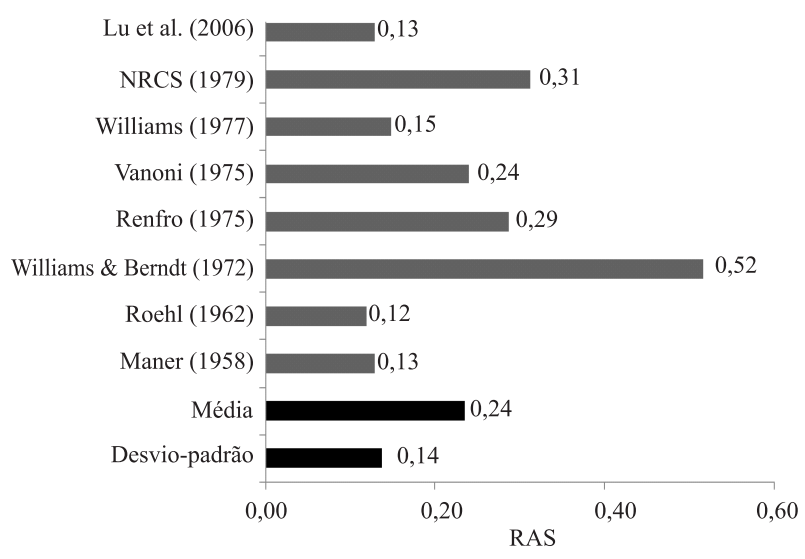

Figura 4. Relação de aporte de sedimento (RAS) para a bacia do ribeirão Pipiripau, usando as oito equações do quadro 1.

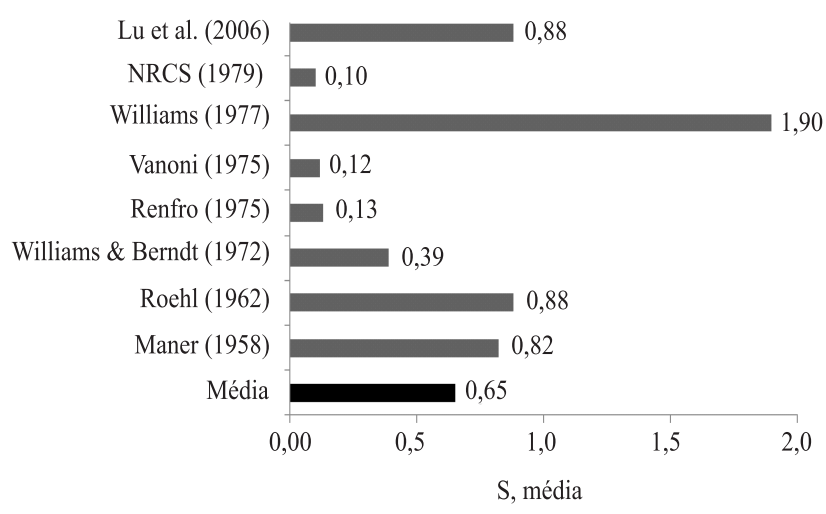

Figura 5. Sensibilidade relativa média das equações de RAS às suas variáveis.

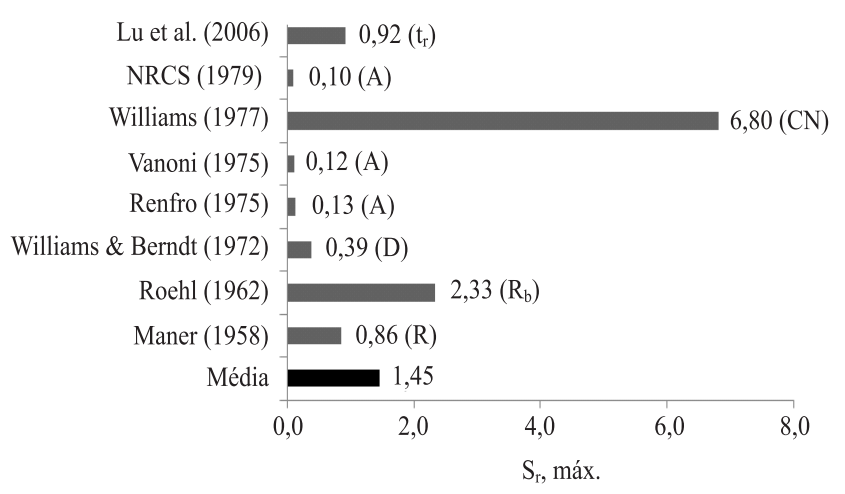

Figura 6. Sensibilidade relativa máxima das equações de RAS às suas variáveis, indicando a variável responsável (ver descrição das variáveis no Quadro 1).

Observa-se que, em média, o modelo mais sensível a perturbações nas variáveis independentes da RAS é o de Williams (1977), com $\mathrm{S}_{\mathrm{r}}=1,90$ (Figura 5). Isso significa que um erro médio de $10 \%$ na estimativa de suas variáveis representaria um erro médio de $19 \%$ na estimativa da RAS, com elevado potencial de propagação de erro. Por sua vez, a equação com o menor valor de $\mathrm{S}_{\mathrm{r}}$ médio $(0,12)$ foi a de Vanoni $(1975)$, 
indicando que um erro médio de $10 \%$ na variável independente causaria um erro de apenas $1,2 \%$ em RAS. Em média, a sensibilidade relativa para as oito equações foi de 0,65 .

Uma análise da figura 6, entretanto, indica que algumas equações apresentaram sensibilidade elevada a algumas de suas variáveis. Esse é o caso da equação de Williams (1977), em que 10 \% de variação em CN causaria variação de $68 \%$ em RAS, o que indica alta instabilidade da equação. No caso da equação de Roehl (1962), a variável que implicou maior sensibilidade foi $R_{b}\left(S_{r}=2,33\right)$.

Conclui-se que as equações de RAS mais estáveis (menor $\mathrm{S}_{\mathrm{r}}$ médio) são as de Vanoni (1975), Renfro (1975) e NRCS (1979), sendo as mais instáveis (maior $\mathrm{S}_{\mathrm{r}}$ máximo) as de Williams (1977) e Roehl (1962).

Implicações do uso de RAS em programas de pagamento por serviço ambiental em bacias

Partindo-se dos valores de RAS calculados para a bacia do ribeirão Pipiripau com as oito equações de RAS supramencionadas, e supondo redução da erosão total na bacia de $\Delta \mathrm{E}=117.500 \mathrm{t}^{\mathrm{ano}} \mathrm{o}^{-1}$, foram obtidas as reduções em $\Delta \mathrm{Y}$ usando os valores de RAS calculados com as oito equações.

De acordo com a figura 7, a redução no aporte de sedimento no exutório da bacia do ribeirão Pipiripau variaria de 14 a 61 mil t ano ${ }^{-1}$, dependendo da equação de RAS usada. Usando-se o valor médio de RAS $(0,24)$, haveria redução de aporte de sedimento anual de 28 mil t. Novamente, a equação que mais se aproximou do valor médio foi a de Vanoni (1975).

$\mathrm{O}$ desvio-padrão de $\Delta \mathrm{Y}\left(16 \mathrm{mil} \mathrm{t}_{\mathrm{t}} \mathrm{ano}^{-1}\right)$ foi elevado, sugerindo que, dependendo do tipo de equação de RAS, haveria elevada incerteza na estimativa da redução da produção de sedimento. Isso tem implicações importantes se a RAS for usada na estimativa do serviço ambiental de programas conservacionistas em bacias hidrográficas.

Para que essa incerteza seja reduzida, é importante que haja um esforço de monitoramento da erosão e do

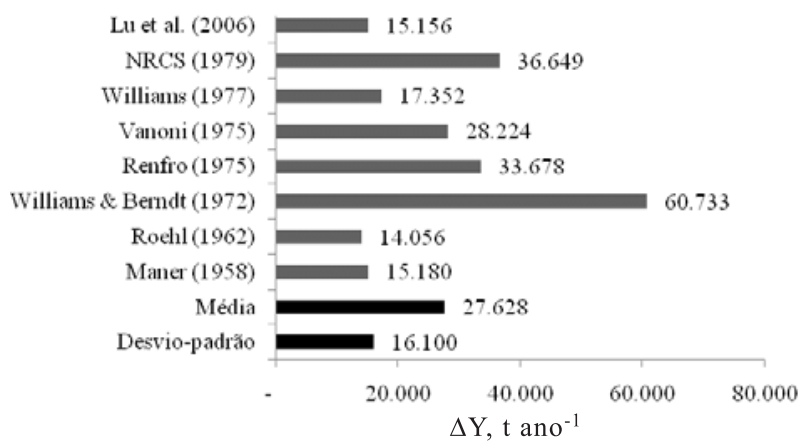

Figura 7. Reduções na produção de sedimento anual $(\Delta \mathrm{Y})$ em função de redução de 50 \% na erosão total na bacia do ribeirão Pipiripau. aporte de sedimento nas bacias, antes, durante e depois da implantação de programas conservacionistas. Essas informações, por sua vez, obtidas de uma maneira sistemática, poderão jogar um pouco de luz sobre o ainda obscuro processo de transferência do sedimento no interior das bacias hidrográficas.

\section{CONCLUSÕES}

1. A relação de aporte de sedimento (RAS), calculada para a bacia do ribeirão Pipiripau usando oito diferentes equações da literatura, apresentou amplitude significativa, variando de 0,12 a 0,52 , com média de 0,24 e desvio-padrão de 0,14.

2. Com o auxílio do SIG, foi relativamente fácil obter as variáveis fisiográficas usadas na estimativa da RAS para a bacia, exceto o número-curva, obtido por meio da calibração com um modelo chuva-vazão, em estudo anterior.

3. Além da grande variação observada nos valores calculados da RAS, algumas equações apresentaram alta sensibilidade às variáveis independentes, destacando-se as de Williams (1977) e Roehl (1962). Nestas duas equações, um erro de $10 \%$ na estimativa de $\mathrm{CN}$ e de $\mathrm{R}_{\mathrm{b}}$ resultaria em erro de $68 \%$ e $23 \%$ na RAS, respectivamente. As outras seis equações tiveram um comportamento mais estável nesse aspecto.

4. Quando usadas para estimar a redução do aporte de sedimento anual na bacia do Pipiripau em função de uma redução de $50 \%$ na erosão total, as oito equações de RAS também mostraram resultados bastante diversos, variando de 14 a 61 mil t ano $^{-1}$, com uma média de 28 mil t ano ${ }^{-1}$. Essa variação em $\Delta \mathrm{Y}$ pode dificultar a estimativa do serviço ambiental relativo à redução da sedimentação nas bacias em programas conservacionistas.

\section{LITERATURA CITADA}

CAESB. Plano de Proteção Ambiental da Bacia do Ribeirão Pipiripau - Diagnóstico ambiental. Brasília, 2001. 89p.

CHAVES, H.M.L. Análise global de sensibilidade dos parâmetros da equação universal de perda de solos modificada (MUSLE). R. Bras. Ci. Solo, 15:345-350, 1991.

CHAVES, H.M.L. \& PIAU, L.P. Efeito da variabilidade da precipitação pluvial e do uso e manejo do solo sobre o escoamento superficial e o aporte de sedimento de uma bacia hidrográfica do Distrito Federal. R. Bras. Ci. Solo 32:333-343, 2008.

CHAVES, H.M.L.; BRAGA, B.; DOMINGUES, A.F. \& SANTOS, D.G. Quantificação dos benefícios ambientais e compensações financeiras do Programa do Produtor de Água/ANA: I. Teoria. R. Bras. Rec. Hídricos, 9:5-14, $2004 a$. 
CHAVES, H.M.L.; BRAGA, B.; DOMINGUES, A.F. \& SANTOS, D.G. Quantificação dos benefícios ambientais e compensações financeiras do Programa do Produtor de Água/ANA: II. Aplicação. R. Bras. Rec. Hídricos, 9:15-21, $2004 \mathrm{~b}$.

CHOW, V.T.; MAIDMENT, D.R. \& MAYS, L.W. Applied hydrology. New York, Mc Graw-Hill, 1988. 572p.

COMITE DA BACIA HIDROGRÁFICA DOS RIOS PIRACICABA, CAPIVARI E JUNDIAÍ-PCJ. Disponível em:<http://www.comitepcj.sp.gov.br/comitespcj.htm>

KIRPICH, P.Z. Time of concentration of small agricultural watersheds. Civil Eng., 10:362, 1940.

LIMA, J.E.F.W.; SANTOS, P.M.C.; CHAVES, A.G.M. \& SCILEWSKI, L.R. Diagnóstico do fluxo de sedimentos em suspensão na bacia do rio São Francisco. Brasília, ANEEL/ANA/Embrapa, 2001. 108p.

LU, H.; MORAN, C.J. \& PROSSER, I.P. Modeling sediment delivery ratio over the Murray Darling basin. Environ. Modelling Software, 21:1297-1308, 2006.

MAGALHÃES, P.C. Hidrologia superficial. In: RAMOS, F., ed. Engenharia hidrológica. Rio de Janeiro, UFRJ/ABRH, 1989. p.201-289.

MANER, S.B. Factors affecting sediment delivery rates in the Red Hills physiographic area. Trans. Am. Geoph. Union, 39:669-675, 1958.

McCUEN, R.H. \& SNYDER, W.M. Hydrologic modeling: Statistical methods and applications. New York, PrenticeHall, 1986. 550p.

OUYANG, D. \& BARTHOLIC, J. Predicting sediment delivery ratio in Saginaw Bay watershed. In: NATIONAL ASSOCIATION OF ENVIRONMENTAL PROFESSIONALS CONFERENCE, 22., Orlando, 1997. Proceedings... Orlando, 1997. p.659-671.

RENFRO, G.W. Use of erosion equations and sediment delivery ratios for predicting sediment yield, in present and prospective technology for predicting sediment yield and sources, - Washington, USDA, 1975. p.33-45. (USDA ARSS-40)
ROEHL, J.E. Sediment source areas, delivery ratios, and influencing morphological factors. Wallingford, IAHS, 1962. p.202-213. (Publication, 59)

SILVA, V.C. Erosão atual, erosão potencial e aporte de sedimento na bacia do rio Paracatu. Brasília, Instituto de Geociências, UnB, 2001, 108 p. (Tese de Doutorado)

SOUZA, C.F.; DORNELES, A.M.; ACIOLLY, L.A. \& MERTEN, G. Comparação entre estimativa de produção de sedimentos na bacia do r. Potiribu. In: ENES, 7., Porto Alegre, 2006. Anais... Porto Alegre, 2006. 20p.

USDA-NRCS. Sediment sources, yields, and delivery ratios. Washington, National Engineering Handbook, Section 3-Sedimetation. 1979. 120p.

VANONI, V.A. Sedimentation engineering. Reston, ASCE Manuals \& Report on Eng., 1975. 405p. (Practice, 54)

WALLING, D.E. The sediment delivery problem. J. Hydrol., 65:209-237, 1983.

WALLING, D.E. Measuring sediment yield from river basins. In: LAL, R., ed. Soil erosion research methods. Ankeny, SWCSA, 1988. p.39-74.

WILLIAMS, J.R. Sediment delivery ratios determined with sediment and runoff models. In: EROSION AND SOLID MATTER TRANSPORT IN INLAND WATERS: SYMPOSIUM, Paris, 1977. Proceedings... Paris, 1977. p.168-179. (Publ. IAHS, 122)

WILLIAMS, J.R. \& BERNDT, H.D. Sediment yield computed with universal equation. Proc. Am. Soc. Civ. Eng., J. Hydrol. Div., 98:2087-2098, 1972.

WISCHMEIER, W.H. \& SMITH, D.D. Predicting rainfall erosion losses: A guide for conservation planning. Washington, 1978. 57p. (USDA Handbook, 537)

YOUNG, R.A. Determining the economic value of water. Washington, RFF, 2005. 356p. 